\title{
Charge fluctuations and their effect on conduction in biological ion channels
}

\author{
D.G. Luchinsky ${ }^{1,2}$, R. Tindjong ${ }^{1}$, I. Kaufman ${ }^{3}$, \\ P.V.E. McClintock ${ }^{1}$ and R.S. Eisenberg ${ }^{4}$ \\ ${ }^{1}$ Department of Physics, Lancaster University, Lancaster, LA1 4YB, UK \\ ${ }^{2}$ NASA Ames, Mail stop 269-3, Moffett Field, CA, 94035, USA. \\ ${ }^{3}$ The Russian Research Institute for Metrological Service, \\ Gosstandart, Moscow, 119361, Russia \\ ${ }^{4}$ Department of Molecular Biophysics and Physiology, Rush Medical College, \\ 1750 West Harrison, Chicago, IL 60612, USA \\ E-mail: r.tindjong@lancaster.ac.uk
}

\begin{abstract}
The effect of fluctuations on the conductivity of ion channels is investigated. It is shown that modulation of the potential barrier at the selectivity site due to electrostatic amplification of charge fluctuations at the channel mouth exerts a leading-order effect on the channel conductivity. A Brownian dynamical model of ion motion in a channel is derived that takes into account both fluctuations at the channel mouth and vibrational modes of the wall. The charge fluctuations are modeled as a shot noise flipping the height of the potential barrier. The wall fluctuations are introduced as a slow vibrational mode of the protein motion that modulates ion conductance both stochastically and periodically. The model is used to estimate the contribution to the conductivity of ion channels coming from the electrostatic amplification of charge fluctuations.
\end{abstract}

\section{Introduction}

Ion transport through the channels in cellular membranes underlies all electrical signal transduction and processing by living organisms. Accordingly ion channels, being natural nanotubes, control a vast range of biological functions in health and disease. The understanding of their structure-properties relationship is the subject of intensive, ever-growing, fundamental and applied research in biology, physics, and nanotechnology $[1,2]$. A central problem in studies of ion permeation through biological membrane channels is to understand how channels can be both highly selective between alike ions and yet still conduct millions of ions per second [3]. Indeed, selectivity between ions of the same charge implies that there exists a deep potential well for conducting type ions at the selectivity site of the channel. On the other hand such channels can pass up to $10^{8}$ ions per second [4] corresponding to almost free diffusion.

Significant progress has been made towards an understanding of this problem over the last few decades. In particular, the molecular structure of the KcsA potassium 
channel [5] that discriminates between $\mathrm{Na}^{+}$and $\mathrm{K}^{+}$was determined by crystallographic analysis. Furthermore, by detecting the size of the structural fluctuations [6] and conformational changes [7], it has become possible to provide the experimental information needed for molecular modeling of the dynamical features of the observed selectivity and gating $[8,9]$. In particular, the minimum radius of the selectivity filter in $\mathrm{KcsA}$ is $\sim 0.85 \AA$, which is to be compared with $1.33 \AA$ for the ionic radius of $\mathrm{K}^{+}$, suggesting that flexibility of the filter is coupled to ionic translocation [10]. It has therefore become apparent that fluctuations in the channel walls play a fundamental role in maintaining high conductivity in highly selective channels (see also Elber [11]).

Another important source of modulation of the electrostatic potential identified in earlier research $[12,13]$ relates to the interaction of the ion in the channel with charge fluctuations in the bath solutions. The effect of current fluctuations and noise on the channel entrance rates and on the channel conductivity was also considered in $[14,15]$. It becomes clear that fluctuations of the electrostatic potential within ion channels induced by various sources may provide a key to the solution of the central problems of permeation and selectivity. Models of such fluctuations have thus a central topic of research on the permeability of ion channels. It is important to note that dynamical models of ion motion in the channel can also provide a link between studies of the permeability of open channels and channels gating. Notwithstanding recent advances, theoretical modeling of the dynamical features of ion channels is still in its infancy. In particular, little is yet known about the relative importance of the different dynamical mechanisms and sources of fluctuations in the ion channels.

In our earlier work we have started to develop a dynamical model $[16,17,18$, $19,20,21]$ of ionic conductivity through open channels. It takes into account the coupling of ion motion to vibrations of the wall $[16,17]$ and to charge fluctuations at the channel mouth $[19,20]$. Our goal is to derive a self-consistent model that allows for analytical estimation of the potential barrier at the selectivity site and for the effects of fluctuations on the conductivity of the channels. The starting point of our approach is a self-consistent quasi-analytical solution of the Poisson and Nernst-Planck equations in the channel, and in the bulk [21], allowing for accurate estimation of the current-voltage characteristics of ion channels [22] (see also $[23,24,25,26,27]$ ). The electrostatic channel potentials resulting from these estimates can be further used to estimate the relative contribution to the channel conductivity from the different sources of fluctuations.

In this paper we introduce a model of ion permeation that takes into account the dynamical effect of the charge fluctuations through the resultant shot noise, and we demonstrate that the latter has a leading-order effect on the transition probabilities. We show that charge fluctuations at the channel mouth can be modeled as a flipping of the electrostatic potential at the selectivity site, which fluctuates between two maximum values at a rate corresponding to the random arrivals of ions at the channel mouth. The theoretical framework developed will allow us in the future to include into the model the modulation of the potential at the selectivity site due to hydration effects inside the 
channel.

A model of 3D Brownian dynamics simulation of ions in the bulk and inside the channel is described in Sec. 2. Using results of the 3D simulations in the bulk we present in Sec. 3 a reduced model of an ion moving in the channel and interacting with the wall vibrational modes and with charge fluctuations at the channel entrance. The model uses the channel potential derived from a self-consistent solution of the Poisson equation and the flipping rates of the potential barrier obtained from simulations of Brownian motion of ions in the bulk. In Sec. 4, we estimate analytically the mean first passage time of the channel. These estimates are based on the assumption that barrier-crossing and barrier-fluctuations are correlated for a general form of the potential barrier [28]. The mean first passage time is calculated as a weighted sum of the escape time $\tau_{-}$over the low barrier $\Delta E_{0}$, and the escape time $\tau_{+}$over the high barrier $\Delta E_{1}$. The results are compared with single-channel data in Sec. 5. Conclusions are drawn and future directions of research are outlined in Sec. 6.

\section{Brownian dynamics simulations in the bulk and inside the channel}

We consider the following Brownian dynamical model of the ion permeation (see sketch in the Fig. 1 (left)). The system is comprised of three compartments of equal size. The middle block represents the protein, through which there is a cylindrical hole approximating the open channel. To model the ion's coupling to the vibrational modes of the channel, we introduce a moving segment of the protein wall that is elastically bound to the wall. The moving segment is charged and represents the selectivity site. The dynamics of the ions in the bath and channel, and of the moving segment, are modeled using Brownian dynamics (BD) simulations, see eqs. (2)-(5) coupled to the Poisson equation (1).

$$
\begin{aligned}
& -\nabla \cdot(\varepsilon(\vec{r}) \nabla \phi(\vec{r}))=\rho(\vec{r}), \\
& m_{i} \ddot{\vec{x}}_{i}+m_{i} \gamma_{i} \dot{\vec{x}}_{i}=\vec{F}_{C, i}+\vec{F}_{s r, i}+\vec{F}_{H, i}+\sqrt{2 m_{i} \gamma_{i} k_{B} T} \vec{\xi}_{i}(t), \\
& m_{j} \ddot{\vec{x}}_{j}+m_{j} \gamma_{j} \dot{\vec{x}}_{j}=\vec{F}_{C, j}+\vec{F}_{s r, j}+\vec{F}_{H, j}+\sqrt{2 m_{j} \gamma_{j} k_{B} T} \vec{\xi}_{j}(t), \\
& m \ddot{x}+m \gamma \dot{x}=F_{C}+F_{c h}+F_{i w, i} \sin (\beta)+\sqrt{2 m \gamma k_{B} T} \xi(t), \\
& M \delta \ddot{R}+M \Gamma \delta \dot{R}+K \delta R=F_{i w, M} \cos (\beta)+\sqrt{2 M \Gamma k_{B} T} \nu(t)
\end{aligned}
$$

Here $\vec{r}_{i j}=\vec{x}_{i}-\vec{x}_{j}, r_{i j}=\left|\vec{r}_{i j}\right|$; and $m_{i}, \vec{x}_{i}$ and $q_{i}, m_{i} \gamma_{i}$ and $\sqrt{2 m_{i} \gamma_{i} k_{B} T} \vec{\xi}_{i}(t)$ are the mass, position, charge, friction coefficient and the stochastic force of or on the $i$ th ion. The distance between ions $i$ and $j$ is $r_{i j}$. For simplicity, we restrict the analysis to two types of ion: the index $i$ will correspond to $\mathrm{Na}^{+}$, while index $j$ corresponds to $\mathrm{Cl}^{-}$. The motion of the charged residual of mass $M$ at the selectivity site is characterized by the displacement $\delta R$ in the vertical direction from the equilibrium position $R$ and an elastic force $\propto K \delta R$. Note that, in general, values of the effective mass and diffusion constant $D$ of the ion moving within the channel may deviate from the corresponding 

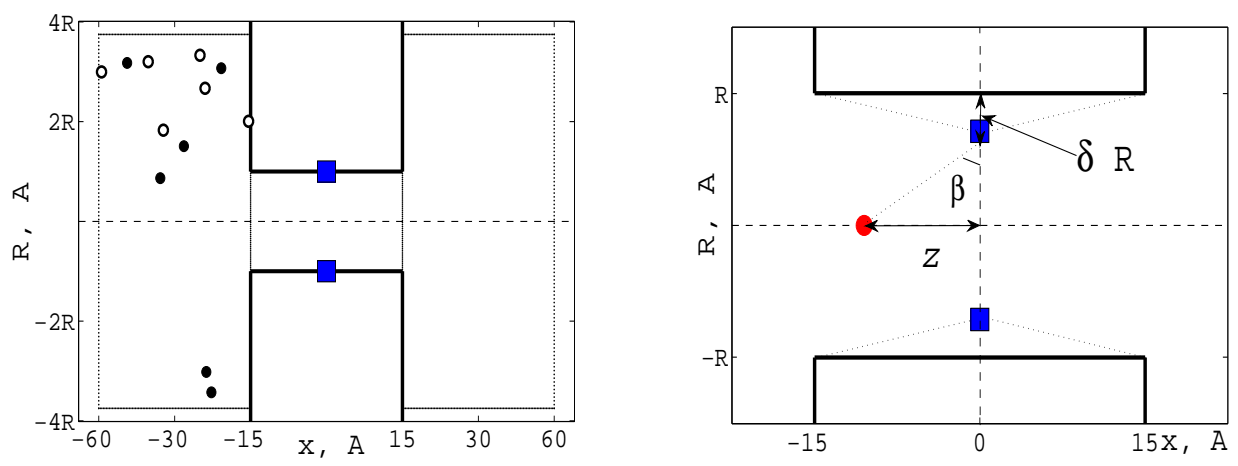

Figure 1. Left: Sketch of the model. The middle block represents the protein, through which there is a cylindrical hole approximating the open ion channel. The moving charged segment of the protein wall, assumed elastically bound to the wall, is shown by the filled squares. Negative ions are shown by the filled circles, and positive ions by the open circles. Right: Sketch of an ion moving along the channel axis. The conducting ion is shown by red circle. The difference between ion coordinate $x$ and location of the selectivity site $x_{s s}$ is $z$. The vertical displacement of the selectivity site is $\delta R$. The angle between a line connecting the ion with the selectivity site and the vertical axis is $\beta$.

values in bulk due to the nontrivial effect of hydration in the channel. Coupling between the motion of the ion in the channel and the normal mode of the wall oscillations is accounted for by the term $F_{i w}$ corresponding to the Coulomb interaction between ion and charge at the selectivity site. Retaining only the axial component of the force for the ion motion in the channel, and the radial component of $F_{i w}$ for the oscillating wall, we have

$$
F_{i w, i}=\frac{Q q_{j}}{4 \pi \epsilon \epsilon_{0} d^{2}} \frac{z}{d}, \quad F_{i w, M}=\frac{Q q_{j}}{4 \pi \epsilon \epsilon_{0} d^{2}} \frac{\delta R}{d} .
$$

Here $d=\sqrt{\left((R+\delta R)^{2}+z^{2}\right)}, z=x-x_{s s}$ where $x$ is the coordinate of the ion in the channel, measured along the channel axis, and $x_{s s}$ is the location of the selectivity site. An additional coupling of the ion motion to fluctuations of the channel wall is effected via modulation of the channel potential by the moving wall. This is taken into account through the term $F_{c h}$ in eq. (4). The ions in the bulk (see eqs. (2), (3)) do not feel the channel potential. Instead, their motion is governed by the Coulomb interaction $F_{C}$, by the short-range interaction $F_{s r}$, and by hydration forces $F_{H}[3]$

$$
\begin{aligned}
& \vec{F}_{C, i}=\sum_{j=1}^{N} \frac{q_{i} q_{j}}{4 \pi \varepsilon \varepsilon_{0} r_{i j}^{2}} \frac{\vec{r}_{i j}}{r_{i j}}, \quad \vec{F}_{s r, i}=\sum_{j=1}^{N} \frac{9 U_{0} R_{c}^{9}}{r_{i j}^{10}} \frac{\vec{r}_{i j}}{r_{i j}}, \\
& \left.\vec{F}_{H, i}=\sum_{j=1}^{N} A U_{0} \exp \left(\frac{R_{i j}-r_{i j}}{a_{e}}\right) \sin \left(2 \pi \frac{R_{i j}-r_{i j}}{a_{w}}-\alpha\right)\right] \frac{\vec{r}_{i j}}{r_{i j}},
\end{aligned}
$$

where $A=\sqrt{1+\left(\frac{a_{w}}{2 \pi a_{e}}\right)^{2}}$ and $\alpha=\arctan \left(\frac{a_{w}}{2 \pi a_{e}}\right)$.

The effect of the surroundings is taken into account by the average frictional force with friction coefficient $m_{i} \gamma_{i}$ and a stochastic force $\sqrt{2 m_{i} \gamma_{i} k_{B} T} \vec{\xi}_{i}(t)$. The addition of the pairwise repulsive $1 / r^{9}$ soft-core interaction potential ensures that ions of opposite 
Table 1. Parameters used in the calculation of the short range ion-ion interaction with hydration

\begin{tabular}{|l|r|r|r||l|l|}
\hline Ions & $U_{0}\left[\mathrm{k}_{B} \mathrm{~T}\right]$ & $R_{c}[\AA]$ & $R[\AA]$ & $a_{w}[\AA]$ & $a_{e}[\AA]$ \\
\hline $\mathrm{Na}-\mathrm{Na}$ & 0.5 & 3.50 & 3.7 & & \\
$\mathrm{Na}-\mathrm{Cl}$ & 8.5 & 2.76 & 2.53 & 2.76 & 1.4 \\
$\mathrm{Cl}-\mathrm{Cl}$ & 1.4 & 5.22 & 5.42 & & \\
\hline
\end{tabular}

charge, attracted by the inter-ion Coulomb force, do not collide and neutralize each other. $U_{0}$ and $R_{c}$ are respectively the overall strength of the potential and the contact distance between ion pairs. The oscillating part, added to the potential, takes explicit account of the internuclear separation for the two solvents, where $a_{w}$ is the oscillation length, $a_{e}$ is the exponential drop parameter, and $R_{i j}$ is the origin of the hydration force which is shifted from $R_{c}$ by $+0.2 \AA$ for like ions and by $-0.2 \AA$ otherwise [29]. $F_{c h}$ is the dielectric force in the channel, obtained by solving Poisson's equation numerically using finite volume methods (FVM) [30]. We use the Langevin equation to model the collective motion of the atoms forming the channel protein's charged ring located at the selectivity filter. In this way, our analysis is based on the assumption that the movement of structural domains of the channel protein may be described as the motions of independent, elastically-bound Brownian particles [31]. We have included the damping term $M \Gamma \delta \dot{R}$ and the corresponding random force $\sqrt{2 M \Gamma k_{B} T} \nu(t)$, whose amplitude is related to the damping constant via the fluctuation-dissipation theorem. The function $\nu(t)$ is a Gaussian white noise. $Q$ is the total fixed charge on the flexible ring interacting with an ion of charge $q_{j}$ on the channel axis $z . R$ is the channel radius, $\delta R$ is a small variation of the channel radius and $K$ is the elastic constant of the channel protein. In the particular case of the Gramicidin A (GA) channel, the value of the elastic constant is estimated by calculating the root-mean-square deviation (RMSD) of the backbone forming its central part, together with the single ion potential of a $\mathrm{Na}^{+}$ ion as it traverses the channel. Using data from [32], we obtain an elastic constant of $\sim 1.66 \mathrm{~N} / \mathrm{m}$ for a maximum RMSD of $1 \AA$. The GA channel molecular weight $M$ is about $4 k D a=6.64 \times 10^{-24} \mathrm{~kg}$ [33]. The diffusion constant of the protein in the membrane is between $10^{-14}$ and $10^{-16} \mathrm{~m}^{2} / \mathrm{s}$ [34].

The parameters of the ion-ion interaction are presented in Table. 1. Other parameters used in the simulations are:

Dielectric constants: $\varepsilon_{2}=80, \varepsilon_{1}=2$;

Masses $(\mathrm{kg}): m_{N a}=3.8 \times 10^{-26}, m_{C l}=5.9 \times 10^{-26}$;

Diffusion coefficients $\left(\mathrm{m}^{2} \mathrm{~s}^{-1}\right): D_{N a}=1.33 \times 10^{-9}, D_{C l}=2.03 \times 10^{-9}$, (where $D$ is related to the friction coefficient via $\left.D=\frac{k_{B} T}{m \gamma}\right)$;

Ion $\operatorname{radii}(\AA): r_{N a}=0.95, r_{C l}=1.81$;

Temperature (K): $T=298$. 

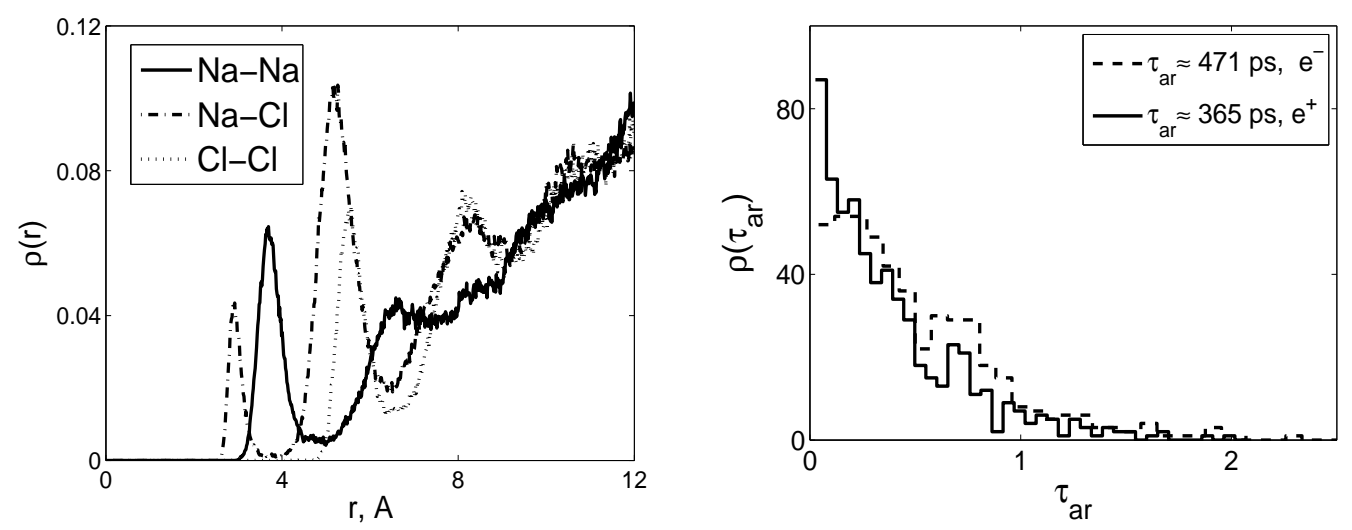

Figure 2. Simulations of Brownian dynamics in the bulk liquid. Left: The number of ions as a function of the distance between ions of different types: $\mathrm{C}=400 \mathrm{mMol}$; Box size: $40 \mathrm{~A}$. Forces included in the simulations were: (i) Coulomb interaction; (ii) short range repulsion; (iii) hydration. Right: The arrival time distribution for positives(solid line) and negative(dashed line) for cylindrical channel of radius $\mathrm{r}=6 \AA$

\section{Reduced model for ion channel conduction}

To derive the reduced model we note that Eqs. (1)-(5) correspond to a many-body problem with widely-varying timescales, ranging from ps (ion fluctuations) to $\mu \mathrm{s}$ (wall vibrations). We further assume that the channel is occupied most of the time by only one ion, and that the transition rate of ions through the channel is mainly determined by escape over the potential barrier at the selectivity site. Thus the effect of the manybody ion dynamics in the bulk on the ion motion in the channel is twofold: (i) delivery of ions to the channel mouth and (ii) modulation of the channel potential by the charge fluctuations at the channel mouth. Under these physiologically plausible assumptions one can separate the ion motion in the channel from the many-body ion dynamics in the bulk. The resulting equations of ion dynamics in the channel coupled to the wall fluctuations can be written as follows

$$
\begin{aligned}
& m \gamma \dot{x}=-\frac{d V(x, t)}{d x}+\sqrt{2 m \gamma k_{B} T} \xi(t), \\
& M \delta \ddot{R}+M \Gamma \delta \dot{R}+K \delta R=F_{i w, M} \cos (\beta)+\sqrt{2 M \Gamma k_{B} T} \nu(t) .
\end{aligned}
$$

Note that the reduced motion of the conducting ion is overdamped, whereas the wall fluctuations are damped. The damped vibrational mode models the relatively slow (on a time scale of ns) motion of the protein of the channel walls that was suggested $[35,12]$ to be essential for the ion transport process.

The potential $V(x, t)$ in our approximation is made up of three main contributions: (i) the potential of Coulomb interaction with ions in the bulk solution $V_{C}$; (ii) the electrostatic potential induced by interaction with the channel protein $V_{c h}$; (iii) the potential of Coulomb interaction with the wall charge at the selectivity site. By an averaging procedure, the effect of multi-ion motion in the bulk solutions is reduced to 

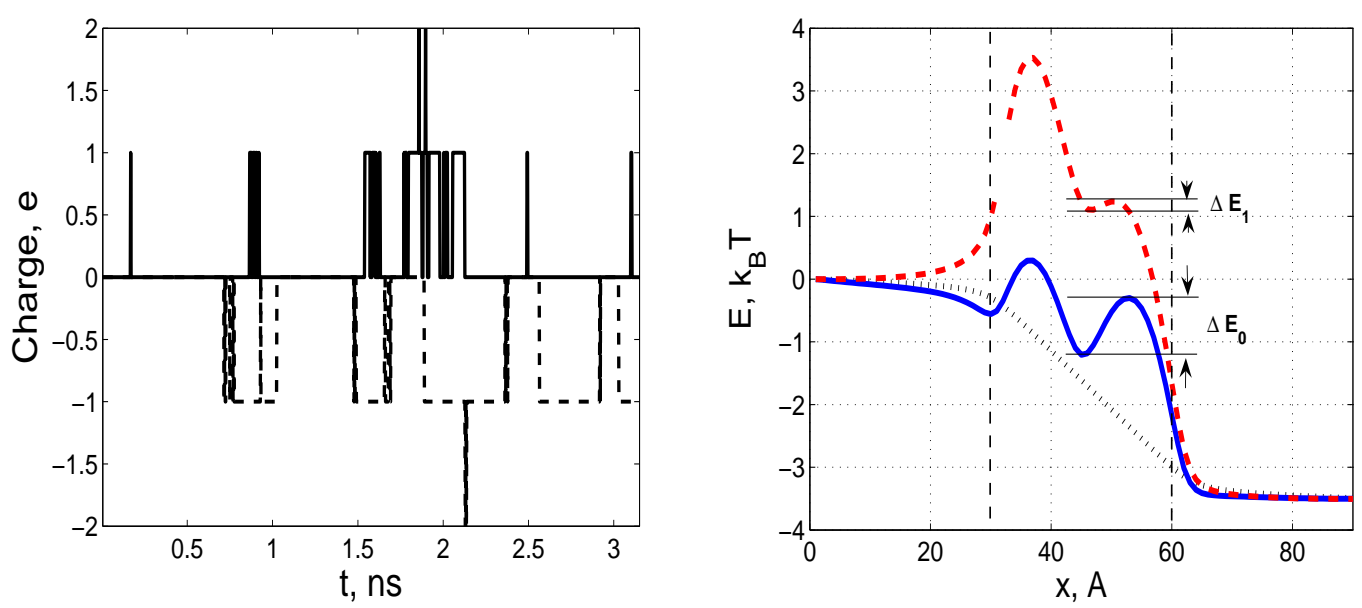

Figure 3. (left) Charge fluctuations at the channel mouth. The positive charge is shown by the solid line. The negative charge is shown by the dashed line. (right) The potential energy profiles as a function of the position of the ion when: the first ion is fixed at the channel mouth (dashed line) and the second is moving along the channel axis. The solid line corresponds to the potential energy on a single ion moving on the channel axis, and the potential energy of the passive channel (dotted line). The vertical dashed lines show the channel entrance and exit. The height of the potential energy barrier seen by a single ion at the selectivity site as it moves from left hand to right hand of the channel is denoted $\Delta E_{0}$. In the presence of a second ion at the channel's left mouth this barrier is reduced to $\Delta E_{1}$.

the Coulomb interaction with ions arriving at the channel mouth. The later process can be viewed as a stochastic Poisson process or as shot noise that modulates the potential barrier for the conducting ion at the selectivity site. Our goal is to estimate analytically the effect of this potential modulation on the channel conductivity, as will be discussed in detail in the next section.

To quantify the effect of multi-ion motion in the bulk on the conducting ion at the selectivity site, we have simulated the ion's Brownian dynamics. The resulting ion-ion distributions in the bulk are shown in Fig. 2 (left). We emphasize that these distributions are very close to those obtained earlier in both $\mathrm{BD}$ simulations [3] and experiments [36]. The arrival time distributions for $\mathrm{Na}^{+}$and $\mathrm{Cl}^{-}$ions at the channel mouth (defined as a cylindrical section of radius $R$ and length $R$ ) obtained in our simulations is shown in Fig. 2 (right). Note that these distributions are exponential for both $\mathrm{Cl}^{-}$and $\mathrm{Na}^{+}$with mean arrival times $\tau^{-}=471 \mathrm{ps}$ and $\tau^{+}=365$ ps respectively. These estimates are in agreement with the theoretical estimates in [37]

$$
\tau_{\text {arrival }}=\frac{1}{2 \pi c D R}
$$

where $c$ is the ion concentration.

The time evolution of the charge in the channel mouth is shown in Fig. 3 (left). It can be seen that the charge at the channel mouth is a Poisson process with the three main states being $+1 e, 0$, and $-1 e$. As a first approximation it is convenient to divide 
the states of the channel potential affected by the charge fluctuations into two effective states: (i) a state of high conductivity, corresponding to $+1 e$; and (ii) two states of low conductivity, corresponding to 0 or $-1 e$ at the channel mouth. In this approximation the effect of having three states of the potential is taken into account by asymmetry of the transition rates between the two effective states. The corresponding transition rates can be estimated as $\alpha^{ \pm}=1 /\left\langle T_{ \pm}\right\rangle$, where $\left\langle T_{ \pm}\right\rangle$are mean residence time of two effective states, giving $\left(\alpha^{+}\right)^{-1}=0.22 \mathrm{~ns}$ and $\left(\alpha^{-}\right)^{-1}=0.38 \mathrm{~ns}$. The occupation probabilities of these two states are 0.36 and 0.64 respectively. The effect of the wall oscillations on the channel potential was estimated earlier [20] and for simplicity the wall will be assumed rigid in the rest of the paper.

To estimate the effect of charge fluctuations on the value of the channel potential we solve the Poisson equation for various positions of the conducting ion along the channel axis in two cases: (i) when there are no other ions at the channel entrances; (ii) when there is one positive ion at the left entrance to the channel. The results of these calculations are shown in Figure. 3(right). The low-conductivity effective state of the channel is shown by the blue solid line and corresponds to the potential barrier $\approx 1 k_{B} T$ at the selectivity site. The high conducting state is shown by the dashed line and corresponds to a potential barrier height $\approx 0.2 k_{B} T$, i.e. practically no barrier state. It can be seen that the charge fluctuations are enhanced in channels of low dielectric constant, resulting in strong modulation of the potential barrier at the selectivity site [20] (see also [38]).

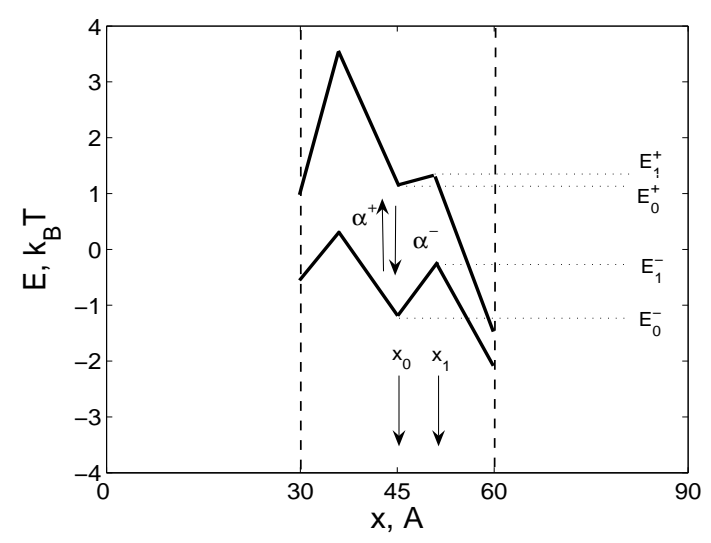

Figure 4. Approximation of the fluctuating potential.

It is therefore possible to build a simple model capable of coupling the motion of ions in the channel to the bath solution. The channel potential becomes

$$
\begin{aligned}
& V(x, t)=\frac{V_{+}+V_{-}}{2}+\frac{V_{+}-V_{-}}{2} \chi(t), \quad \chi(t)= \pm 1, \\
& V_{+}=\frac{\Delta E_{1}}{x_{m}}\left(x-x_{0}\right)+E_{0}^{+}, \quad V_{-}=\frac{\Delta E_{0}}{x_{m}}\left(x-x_{0}\right)+E_{0}^{-},
\end{aligned}
$$

where $\chi(t)$ is a Poisson random force with two transition rates $\alpha_{ \pm}$between the states +1 and -1 . The charge fluctuations at the channel mouth thus result in flipping of the 
potential. Here $\Delta E_{0}=E_{1}^{-}-E_{0}^{-}$and $\Delta E_{1}=E_{1}^{+}-E_{0}^{+}$, with $\Delta E_{0}>\Delta E_{1}$ are respective barriers of the potential in two states, and $x_{m}=x_{1}-x_{0}$.

A direct analogy can be made between the model described by Eq. (6) and the model described by Zürcher [28] whose barrier fluctuation is controlled by a dichotomic noise of zero mean and exponential correlation. An approximation of the fluctuating potential is sketched in Figure. 4. The similarity of the two problems suggests that there is some possibility of semi-analytical estimations of the effect of the charge fluctuations.

\section{Estimation of the mean channel crossing time}

We are interested in the transition of a particle initially trapped at the channel selectivity filter. This corresponds to the motion of the ion across the interval $\left[x_{0}, x_{1}\right]$. The approximate potential in this interval is given by (9). We are interested at the unidirectional current, so there is no backward flow of ions. We assume that on average, the channel is always occupied by a single $\mathrm{Na}^{+}$ion. This arises from the fact that, when an ion is sitting in the middle of the channel, it is almost impossible for a second ion to enter the channel due to the height of the barrier at the left entrance of the channel, as can be seem from Figure. 4.

As a first approximation, we assume that the mean first passage time (MFPT) is only determined by escape. Therefore the MFPT for the channel is expected to be a function of the two times $\tau_{+}$and $\tau_{-}$corresponding to the escape times from the potential minimum in two effective states of the potential. Our derivation follows very closely the earlier discussion by Zürcher [28] with the difference that, here, we are interested in the asymmetric case with two transition rates.

Assuming no back-flow, the quantities $\tau_{ \pm}(x)$ are defined [39] by:

$$
\begin{aligned}
& \frac{-1}{m \gamma} \frac{d V_{+}}{d x} \frac{d \tau_{+}}{d x}+\frac{k_{B} T}{m \gamma} \frac{d^{2} \tau_{+}}{d x^{2}}-\alpha^{+} \tau_{+}+\alpha^{-} \tau_{-}=-p_{+}, \\
& \frac{-1}{m \gamma} \frac{d V_{-}}{d x} \frac{d \tau_{-}}{d x}+\frac{k_{B} T}{m \gamma} \frac{d^{2} \tau_{-}}{d x^{2}}-\alpha^{-} \tau_{-}+\alpha^{+} \tau_{+}=-p_{-},
\end{aligned}
$$

The potential jumps between positive and negative values, with respective rates $\alpha^{+}$and $\alpha^{-} \cdot p_{ \pm}$are the occupation probabilities of these states. We choose a reflecting boundary condition (BC) at the bottom of the barrier $x=x_{0}$ and an absorbing $\mathrm{BC}$ at the top of the barrier $x=x_{1}$,

$$
\frac{d \tau_{ \pm}\left(x=x_{0}\right)}{d x}=0, \quad \tau_{ \pm}\left(x=x_{1}\right)=0
$$

With $\tau_{+}$and $\tau_{-}$specified, the mean exit time of the Brownian particle initially trapped at the selectivity filter $x=x_{0}$ is given by:

$$
\tau=\tau_{+}\left(x_{0}\right)+\tau_{-}\left(x_{0}\right),
$$

Following Zürcher [28], the calculation of $\tau_{ \pm}$is straightforward. We introduce

$$
E=\frac{\Delta E_{1}+\Delta E_{0}}{2}, \quad \Delta=\frac{\Delta E_{1}-\Delta E_{0}}{2},
$$



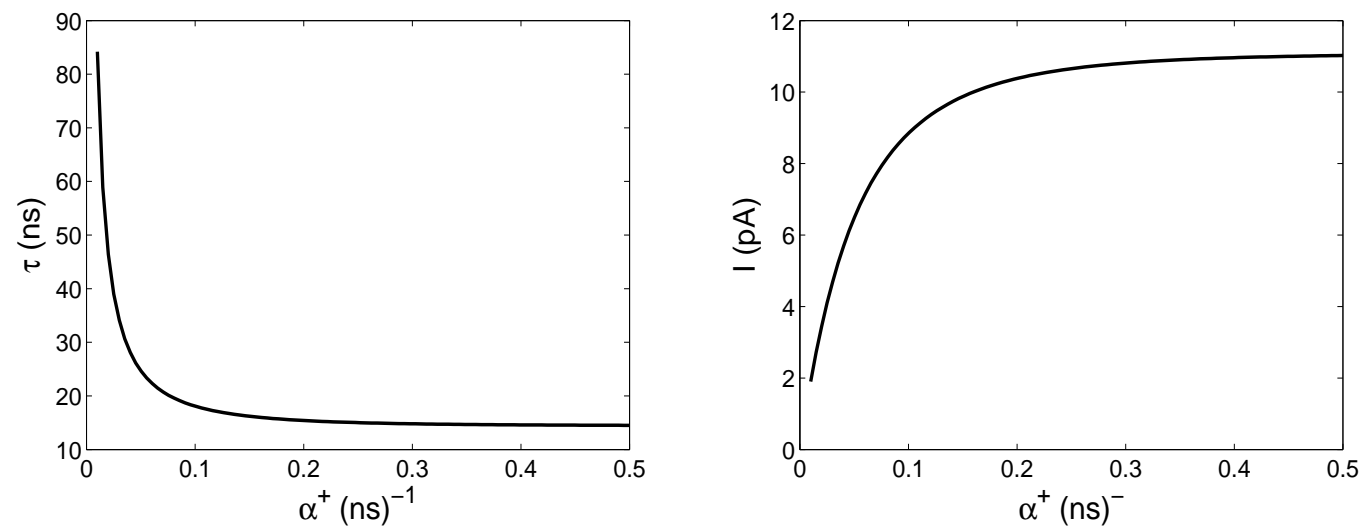

Figure 5. Left: MFPT as a function of $\alpha^{+}$. Right: Current as a function of $\alpha^{+}$.

and

$$
\sigma(x)=\alpha^{+} \tau_{+}(x)+\alpha^{-} \tau_{-}(x), \quad \delta(x)=\alpha^{+} \tau_{+}(x)-\alpha^{-} \tau_{-}(x) .
$$

We find the coupled differential equations

$$
\begin{aligned}
& \frac{-D E}{x_{m} k_{B} T} \frac{d \sigma}{d x}+D \frac{d^{2} \sigma}{d x^{2}}+\left(\alpha^{+} p_{+}+\alpha^{-} p_{-}\right)=\left(\alpha^{+} p_{+}-\alpha^{-} p_{-}\right) \delta+\frac{D \Delta}{x_{m} k_{B} T} \frac{d \delta}{d x}, \\
& \frac{-D E}{x_{m} k_{B} T} \frac{d \delta}{d x}+D \frac{d^{2} \delta}{d x^{2}}-\left(\alpha^{+} p_{+}+\alpha^{-} p_{-}\right) \delta+\left(\alpha^{+} p_{+}-\alpha^{-} p_{-}\right)=\frac{D \Delta}{x_{m} k_{B} T} \frac{d \sigma}{d x},
\end{aligned}
$$

where $D=D_{N a}$ and the boundary conditions,

$$
\sigma\left(x=x_{1}\right)=0, \quad \frac{d \sigma\left(x=x_{0}\right)}{d x}=0, \quad \delta\left(x=x_{1}\right)=0, \quad \frac{d \delta\left(x=x_{0}\right)}{d x}=0 .
$$

We introduce the following scaled dimensionless coordinate,

$$
x=\frac{x_{m} k_{B} T}{E} y .
$$

The model is therefore characterized by the following parameters,

$$
\tau_{0}=2 \frac{\left(x_{m} k_{B} T\right)^{2}}{D E^{2}}, \quad \eta=\frac{\Delta}{E}, \quad \lambda=\frac{\tau_{0}}{2}\left(\alpha^{+} p_{+}+\alpha^{-} p_{-}\right), \quad \beta=\frac{\tau_{0}}{2}\left(\alpha^{+} p_{+}-\alpha^{-} p_{-}\right) .
$$

The coupled differential equations then read,

$$
\begin{aligned}
& -\frac{d \sigma}{d y}+\frac{d^{2} \sigma}{d y^{2}}+\lambda=\beta \delta+\eta \frac{d \delta}{d y}, \\
& -\frac{d \delta}{d y}+\frac{d^{2} \delta}{d y^{2}}-\lambda \delta+\beta=\eta \frac{d \sigma}{d y},
\end{aligned}
$$

and $\sigma(y)$ and $\delta(y)$ are subject to the $\mathrm{BC}$,

$$
\sigma\left(y=y_{1}\right)=0, \quad \frac{d \sigma\left(y=y_{0}\right)}{d y}=0, \quad \delta\left(y=y_{1}\right)=0, \quad \frac{d \delta\left(y=y_{0}\right)}{d y}=0 .
$$

The solution of this system gives,

$$
\delta(y)=\sum_{i=1}^{3} a_{i} \exp \left(q_{i} y\right)+\frac{(\beta-\lambda \eta)}{(\lambda-\beta \eta)}
$$



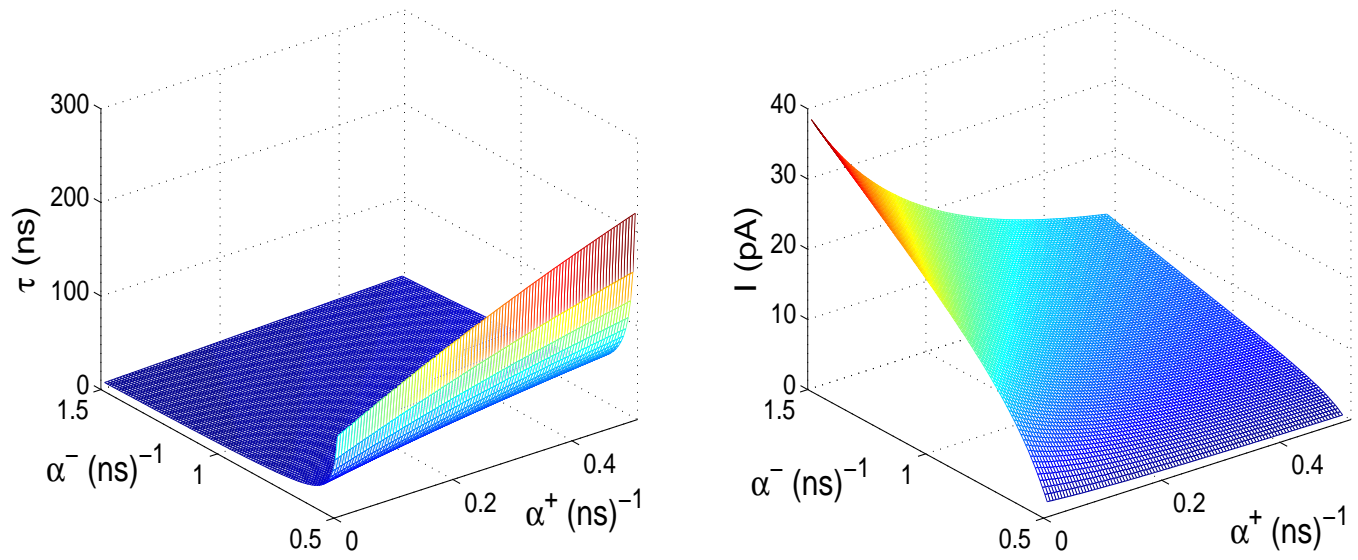

Figure 6. (left) MFPT as function of $\alpha^{+}$and $\alpha^{-}$. (right) current as function of $\alpha^{+}$ and $\alpha^{-}$.

The eigenvalues $q_{i}$, follow from

$$
\left\{\begin{array}{l}
q_{1}+q_{2}+q_{3}=2, \\
q_{1} q_{2}+q_{2} q_{3}+q_{3} q_{1}=1-\lambda-\eta^{2} \\
q_{1} q_{2} q_{3}=-(\lambda-\beta \eta) .
\end{array}\right.
$$

The $a_{i}$ are given as follows,

$$
\begin{aligned}
& a_{1}=-\frac{\beta-\lambda \eta}{D_{s}(\lambda-\beta \eta)}\left(q_{3}\left(q_{2}^{2}-\lambda\right)-q_{2}\left(q_{3}^{2}-\lambda\right)\right) \exp \left(\left(q_{3}+q_{2}\right) y_{0}\right) \\
& -\frac{1}{D_{s}}\left(\frac{\lambda(\beta-\lambda \eta)}{\lambda-\beta \eta}-\beta\right)\left(q_{3} \exp \left(q_{3} y_{0}+q_{2} y_{1}\right)-q_{2} \exp \left(q_{3} y_{1}+q_{2} y_{0}\right)\right) .
\end{aligned}
$$

Here $a_{2}$ and $a_{3}$ are determined by cyclic permutation of the indices of the $q_{i}$ from $a_{1}$, and $D_{s}$ is given by

$$
D_{s}=q_{1} q_{2}\left(q_{2}-q_{1}\right)\left(q_{3}-1\right) \exp \left(\left(q_{1}+q_{2}\right) y_{0}+q_{3} y_{1}\right)+\text { cycl.perm. }
$$

At $y_{0}$, we have:

$$
\begin{aligned}
& \sigma\left(y_{0}\right)=\frac{1}{\eta} \sum_{i=1}^{3} a_{i}\left[\left(-1+q_{i}-\frac{\lambda}{q_{i}}\right) \exp \left(q_{i} y_{0}\right)-\left(q_{i}-\frac{\lambda}{q_{i}}\right) \exp \left(q_{i} y_{1}\right)\right] \\
& -\frac{(\beta-\lambda \eta)}{\eta(\lambda-\beta \eta)}+\left[\frac{\lambda}{\eta} \frac{(\beta-\lambda \eta)}{(\lambda-\beta \eta)}-\frac{\beta}{\eta}\right]\left(y_{1}-y_{0}\right)
\end{aligned}
$$

Combining Eq. (13 and 15), the mean exit time for the Brownian particle follows:

$$
\tau=\frac{\sigma\left(y_{0}\right)+\delta\left(y_{0}\right)}{2 \alpha^{+}}+\frac{\sigma\left(y_{0}\right)-\delta\left(y_{0}\right)}{2 \alpha^{-}} .
$$

The effect of shot noise on ion channel conduction is related to the arrival of ions at the channel's mouth. We calculated the channel MFPT as a function of the two flipping rates. Since the channel has a high affinity for the ions and therefore slows them down, we have used a smaller ion diffusion coefficient inside the channel equal to $1.33 \times 10^{-10}$ 
$\mathrm{m}^{2} \mathrm{~s}^{-1}$. The results of the calculations are shown in Figs. 5 and 6 . It is clear from Fig. 5 that the particle takes longer to cross $\Delta E_{0}$ as compared to $\Delta E_{1}$. There is a fast drop in MFPT as the flipping rate $\alpha^{+}$increase. The current $I=e / \tau$ is also presented as function of $\alpha^{+}$. A more general view of the dependance of the MFPT and the current on the two rates is shown in Fig. 6. We emphasize that the transition rates obtained are essentially non-equilibrium. In particular, a current saturation effect can be observed as $\alpha^{+}$increases for a wide range of parameters. This is in accord with experimental the observation [33] of current saturation at high concentrations.

\section{Comparison with experiment}

\section{Conclusion}

In summary, we have introduced a Brownian dynamical model of ionic transitions through a channel, taking into account charge fluctuations at the channel mouth and the fluctuations of the channel walls. The statistical properties of the charge fluctuations were reconstructed from 3D Brownian dynamics simulations of multi-ion motion in the bulk solution. It was shown that distributions of ion arrival times at the channel mouth are exponential. It was further shown that these charge fluctuations strongly modulate the potential barrier for the conducting ion at the selectivity site due to amplification of electrostatic interactions in long narrow channels of low dielectric constant. These findings have allowed us to model the mean ion transition time through the channel as an ionic escape from the potential well at the selectivity site induced by thermal fluctuations and by modulation of the height of the barrier by stochastic Poisson processes. The proposed model is a Brownian dynamical model of a "knock-on" mechanism [40,41]. It allows for analytic estimation of transition probabilities in the presence of charge fluctuations, i.e. it allows for analytic estimation of correlations between bulk concentrations and ion currents in charged narrow channels. In particular, it demonstrates the effect of current saturation due to ion concentrations in the bath. The model is of an essentially non-equilibrium character. This last point is worth emphasizing because traditional approaches assume equilibrium rates of hopping between the sites.

The model takes account of the wall fluctuations. This latter feature is very important e.g. for an analysis of the tightly correlated motion at the selectivity site, of the type discovered in KcsA [42]. Our model allows for analytic estimation of transition rates in the presence of oscillations of channel walls, using our earlier results [43] on escape from periodically driven potentials based on application of the logarithmic susceptibility [44], as will be discussed in detail elsewhere. Such an escape process, assisted by the periodic modulation of the potential barrier by the wall oscillations, can result on its own in selectivity between alike ions due to the difference in their diffusion coefficients $[45,46]$. However, ultimately the selectivity of the channel has to be incorporated into the model by taking into account the effects of hydration [47] (see 
also [20] for a discussion of how hydration effect can be incorporated in our model). It is also worth mentioning that a model taking into account wall fluctuations may account for dissipation of energy in the channel, for self-induced acceleration of the transition rate of the ion through the channel, and for coupling of the ion motion to the channel gating mechanics. Indeed, in this model, part of the energy induced by a very strong Coulomb interaction between the charged site at the channel wall and the moving ion is stored as the energy of vibrational modes. The latter energy is only partially dissipated by the protein phonon modes. The remaining energy can now be used to modulate the potential barrier for the moving ion in a self-consistent manner to accelerate its transition through the channel. It can also be used to assist the conformational changes that lead to channel gating.

The work in progress contains a plethora of unsolved problems. The immediate extension of the proposed model will be to include more then two levels for the potential at the selectivity site, taking into account positive and negative (or zero) charge at each mouth of the channel. The model can be further refined by including estimates of the return times corresponding to a return of the ion in the channel to the initial bulk solution.

Following the discussion above, we formulate the following general unsolved problems in ion channels:

(i) The role of the membrane fluctuations.

(ii) The role of the hydration potential.

(iii) The role of additional binding sites outside the selectivity filter.

(iv) The energetics of the ion transition including energy relaxation due to the coupling to the protein phonon modes (wall oscillations).

(v) The coupling of the ion-wall interaction to the gating mechanism.

We believe that they can all be be tackled by extending the model introduced in this paper. In each case, noise and dynamical effects seem to play a crucial role that is only just starting to be elucidated.

\section{Acknowledgements}

We are grateful to the Engineering and Physical Sciences Research Council (UK) for financial support, and to A. Stefanovska for valuable discussions

\section{References}

[1] Hille B 1992 Ionic Channel Of Excitable Membranes (Sunderland, MA: Sinauer Associates)

[2] Eisenberg R S 1998 Contemp. Phys. 39 447-466

[3] Corry B, Allen T W, Kuyucak S and Chung S H 2001 Biophys. J. 80 195-214

[4] Jordan P C 2005 IEEE Trans. Nanobiosci. 4 3-9

[5] Doyle D, Cabral J M, Pfuetzer R A, Kuo A, Gulbis J M, Cohen S L, Chait B T and MacKinnon R 1998 Science $\mathbf{2 8 0} 69-77$ 
[6] Zhou Y, Morais-Cabral J H, Kaufman A and MacKinnon R 2001 Nature 414 43-48

[7] Dutzler R, Campbell E B, Cadene M, Chait B T and MacKinnon R 2002 Nature 415 287-294

[8] Corry B 2006 Molecular Biosys. 2 527-535

[9] Noskov S Y, Berneche S and Roux B 2004 Nature 431 830-834

[10] Shrivastava I A, Tieleman D P, Biggin P C and Sansom M S P 2002 Biophys. J. 83 633-645

[11] Elber R, Chen D P, Rojewska D and Eisenberg R 1995 Biophys. J. 68 906-924

[12] Heinemann S H and Sigworth F J 1990 Biophys. J. 57 499-514

[13] Nadler B, Schuss Z, Hollerbach U and Eisenberg R S 2004 Phys. Rev. E 70051912

[14] Bezrukov S M and Vodyanoy I 1995 Nature 378 362-364

[15] Adair R K 2003 Proc. Nat. Acad. Sci. 100 12099-12104

[16] Tindjong R, Applegate A, Eisenberg R S, Kaufman I, Luchinsky D G and McClintock P V E 2004 Fluctuations and Noise in Biological, Biophysical and Biomedical Systems II, SPIE Proc. vol 5467, ed Abbott D, Bezrukov S M Der A, and Sanchez A (Bellingham, WA: SPIE) pp 338-344

[17] Tindjong R, Eisenberg R S, Kaufman I, Luchinsky D G and McClintock P V E 2005 Proc. 18th Intern. Conf. on Noise and Fluctuations, AIP Proc. vol 780, ed Gonzalez T, Mateos J, and Pardo D (Melville, NY: AIP) pp 563-566

[18] Tindjong R 2006 A Self-consistent Approach to the Simulation of Ionic Motion in Open Ion Channels Ph.D. thesis, Lancaster University, Lancaster

[19] Tindjong R, Luchinsky D G, McClintock P V E, Kaufman I and Eisenberg R S 2007 Proc. 19th Intern. Conference on Noise and Fluctuations vol 922 ed Tacano M, Yamamoto Y, and Nakao M (Melville, NY: AIP) pp 647-650

[20] Luchinsky D G, Tindjong R, McClintock P V E, Kaufman I and Eisenberg R S 2007 Noise and Fluctuation in Biological, Biophysical, and Biomedical Systems: D6020-D6020 vol 6602 ed Bezrukov S M (Bellingham, WA: SPIE)

[21] Luchinsky D G, Tindjong R, McClintock P V E, Kaufman I and Eisenberg R S 2007 Noise and Fluctuation in Biological, Biophysical, and Biomedical Systems: E6020-E6020 vol $6602 \mathrm{ed}$ Bezrukov S M (Bellingham, WA: SPIE)

[22] Luchinsky D G, Tindjong R, Kaufman I, McClintock P V E and Eisenberg R S 2008 to be submitted to PRE

[23] Chen D P, Barcilon V and Eisenberg R S 1992 Biophys. J. 61 1372-1393

[24] Elber R, Chen D P, Rojewska D and Eisenberg R 1995 Biophysical Journal 68 906-924

[25] Nonner W and Eisenberg B 1998 Biophys. J. 75 1287-1305

[26] Chen D P, Xu L, Tripathy A, Meissner G and Eisenberg B 1999 Biophys. J. 76 1346-1366

[27] Eisenberg R S 1999 J. Membr. Biol 71 1-24

[28] Zürcher U and Doering C R 1993 Phys. Rev. E. 47 3862-3869

[29] Moy G, Corry B, Kuyucak S and Chung S H 2000 Biophys. J. 78 2349-2363

[30] Ferziger J and Peric M 1996 Computational Method for Fluid Dynamics. (Berlin: Springer)

[31] Läuger P 1985 Biophys. J. 48 369-373

[32] Mamonov A B, Coalson R D, Nitzan A and Kurnikova M G 2003 Biophys. J. 84 3646-3661

[33] Andersen O S, Koeppe R E and Roux B 2005 IEEE Transactions on Nanoscience 4 10-20

[34] Tuszynski J A and Kurzynski M 2003 Introduction to Molecular Biophysics (Florida: CRC Press)

[35] Skerra A and Brickmann J 1987 Biophys. J. 51 977-983

[36] Guardia E, Rey R and Padro J A 1991 J. Chem. Phys 95 2823-2831

[37] Eisenberg R S, Klosek M M and Schuss Z 1995 J. Chem. Phys. 102 1767-1780

[38] Bastug T and Kuyucak S 2003 Biophys. J. 84 2871-2882

[39] Hanggi P and Talkner P 1985 Phys. Rev. A 32 1934-1937

[40] Hodgkin A L and Keynes R D 1955 J Physiol 128 61-88

[41] Berneche S and Roux B 2003 Proceedings of the National Academy of Sciences 100 8644-8648

[42] Kutluay E, Roux B and Heginbotham L 2005 Biophys. J. 88 1018-1029

[43] Luchinsky D G, Greenall M J and McClintock P V E 2000 Phys. Lett. A 273 316-321

[44] Smelyanskiy V N, Dykman M I, Rabitz H and Vugmeister B E 1997 Phys. Rev. Lett. 79 3113-3116 
[45] Marchesoni F 1998 Physics Letters A 237 126-130

[46] Sintes T and Sumithra K 2002 Physica A: Statistical Mechanics and its Applications 312 86-98

[47] Choe S and Robinson R 1998 Neuron 20 821-823 